Editorial

\title{
The needle, or the knife?
}

Dean R Cugley MBBS(Hons), ${ }^{1}$ Xavier Fagan FRANZCO ${ }^{1,2}$

1. Royal Victorian Eye and Ear Hospital, Centre for Eye Research Australia, Australia

2. Austin Hospital, Heidelberg VIC 3084, Australia

Correspondence: Dean Cugley, Royal Victorian Eye and Ear Hospital, Centre for Eye Research Australia, Australia

Contact: Dean.cugley@eyeandear.org.au

Conflict of interest: None

Funding sources: None

This is the author manuscript accepted for publication and has undergone full peer review but has not been through the copyediting, typesetting, pagination and proofreading process, which may lead to differences between this version and the Version of Record. Please cite this article as doi: $10.1111 /$ ceo.13413

This article is protected by copyright. All rights reserved. 
Polypoidal Choroidal Vasculopathy (PCV) remains somewhat of a clinical enigma. It can be seen in both sexes and at varying ages, with a predilection for Asian populations. The first published case series in 1990, described 11 patients with unique features of nodular, polypoidal subretinal lesions with red-orange discolouration, serosanguinous pigment epithelial detachments (PED) and exudative retinal detachments; and was hence proposed as a distinct clinical entity. ${ }^{1}$ PCV can then be further subclassified by their location, or by sequelae into haemorrhagic, exudative or quiescent subtypes. Subretinal fibrosis and retinal atrophy may also be seen.

Advances in multimodal imaging has contributed to PCV becoming an increasingly recognised and managed condition. OCT changes include a PED that may be notched, subretinal hyperreflectivity or 'double layer sign' and 'pachychoroid' or choroidal thickening > 300 microns (well imaged on enhanced depth imaging OCT). Fundus Fluorescein Angiography is typically non-specific with an appearance similar to an occult Choroidal Neovascularisation Membrane (CNVM). This can lead to a potential misdiagnosis of age-related macular degeneration (ARMD) adopted. Indeed, it can be argued that PCV represents a subtype of macular degeneration in some instances. Yet in other instances there are no common features. Many previous papers have included PCV, deliberately or inadvertently, in their cohorts. It should be emphasised that Indocyanine Green Angiography (ICGA) remains the gold standard for the diagnosis if PCV, which typically identifies single or multiple focal hyperfluorescent lesions and adjacent branching vascular network (BVN). ${ }^{2,3}$ OCT Angiography (OCTA) may offer further insights into the study of PCV.

What is evident currently, is that there is a general paucity of information in the medical literature of the epidemiology and pathophysiology of PCV. Only one paper has estimated the incidence at 3 per 1000 in the general northern Chinese 
population, based on OCT and fundus photography parameters. ${ }^{4}$ The pathophysiology of PCV is a topic of much interest and debate, and dynamic choroidal changes and the contribution of inflammatory changes specifically represent area of active, ongoing research. The natural history of PCV is unpredictable. One prospective observational case series study by Uyama et al reported 14 eyes observed regularly and untreated for a 2 year period, of which $50 \%$ had favourable outcomes and 50\% experienced vision loss, with progressive exudate or haemorrhage. ${ }^{5} \mathrm{~A}$ recent retrospective review by Alasil et al of patients from the US and UK reported a $20 \%$ incidence of bilateral disease, variable female predominance and identified poor visual prognostic features of advanced age, worse presenting vision and macular polyp location. ${ }^{6}$ Risk factors for submacular haemorrhage specifically may include pulsatile polyps, cluster-like lesions and systemic anticoagulation. ${ }^{7-9}$

Treatment of PCV focuses primarily on maintaining and improving visual acuity but may have secondary aims including complete anatomic polyp closure and/or regression, which may have an effect on reducing recurrence. Treatments may include thermal laser (reserved for extrafoveal polyps), photodynamic therapy (PDT) and anti-vascular endothelial growth factor (anti-VEGF) injections. ${ }^{2}$ Combination therapy with anti-VEGF and PDT has been evaluated in the recently published RCTs EVEREST II and PLANET studies respectively. $(10,11)$ Surgery is generally reserved for complicated cases, and in particular, subretinal with or without vitreous haemorrhage.

EVEREST II suggested that ranibizumab combined with PDT offered superior visual outcomes than Anti-VEGF monotherapy by around 3 EDTRS letters, required fewer injections (4 vs 7), and had double the polyp closure rate (70\% vs $35 \%$ ) at 12 months. (10) PLANET reported that aflibercept monotherapy was a suitable 
treatment of PCV ( $85 \%$ of patients were dry and inactive at 1 year) and most cases demonstrated a prompt and significant clinical benefit, with few patients $(<15 \%)$ requiring rescue PDT. ${ }^{11} 3$ cases of vitreous haemorrhage were reported in EVEREST II from 322 eyes ( 2 in monotherapy arm and 1 in combination arm), but it is not clear if this was related to submacular haemorrhage and breakthrough or some other cause. (10) Of 318 eyes enrolled in PLANET, only 1 who received rescue PDT lost vision due to subretinal and vitreous haemorrhage in the study eye, which was labelled a PDT-related side effect. (11) It should be noted that the studies had different protocols with EVEREST II implementing PDT at commencement of treatment and PLANET using PDT as a rescue only.

In this issue, Kang and colleagues report a retrospective review of 48 consecutive Korean eyes diagnosed with significant submacular haemorrhage involving the fovea centre secondary to PCV, and describe their outcomes when allocated one of three treatment arms: intravitreal bevacizumab (IVB) alone, IVB with pars plana vitrectomy (PPV), or IVB with pneumatic displacement (PD). (12) Pathways were allocated by the treating specialist and despite the largest group those undergoing IVB monotherapy, baseline VA suggest that those with worse vision were significantly more likely to undergo PPV. Furthermore, all patients reviewed were PDT naïve as mandated by exclusion criteria, indicating all episodes were related to PCV pathogenesis and not a side effect of PDT.

Overall, at 2 years post intervention there was no reported sustained overall difference in BCVA between the three groups. (12) Despite small numbers $(\mathrm{N}=10)$ the potential for significant visual improvement ( 3 line gain or more) may be more likely with pneumatic displacement. Patients undergoing IVB monotherapy required substantially more injections, however this may relate to the threshold and details of the retreatment criteria. 
As difficult as it is to obtain consensus on optimal treatment strategies for macular $\mathrm{PCV}$, it is further complicated by additional treatment options in the presence of haemorrhage. Kang et al ask the question: should we be discussing surgery more frequently in PCV patients with submacular haemorrhage, and undertaking pneumatic displacement in attempt to reach for a significant visual gain? One study of ARMD patients has suggested that visual outcomes may be better for submacular haemorrhages greater than $\mathbf{4 5 0}$ microns in thickness if they are pneumatically displaced compared to sole anti-VEGF monotherapy; ${ }^{13}$ a parameter which may better guide patient selection for intervention. Combining pneumatic displacement with anti-VEGF in PCV has been shown to result in superior visual outcomes and decreased treatment burdens compared to PD alone, ${ }^{14}$ emphasising that surgery should be seen as adjunct to anti-VEGF. Other options such as intravitreal recombinant tissue Plasminogen Activator (ItPA) compared to vitrectomy and subretinal ItPA, have been studied in patients with ARMD with equivalent visual outcomes, ${ }^{15}$ but such approaches have not universally been adopted.

Further study to identify which patients are most likely or unlikely to benefit from surgical intervention, along with development of less invasive strategies and techniques would provide more valuable insight into our currently relatively crude decision making in the management of submacular haemorrhage due to PCV. At this stage, all suggested treatments have a role in managing PCV and its spectrum of manifestations, with the exact parameters which determine intervention yet to be defined. Case series such as these add weight to management strategies for this increasingly recognized, unpredictable and occasionally unforgiving condition. The inherent flaws and limitations of case series are well known. However, in the absence of large, multi-centre, randomized controlled trials, such evidence is the pillar on which we must base clinical decision making. 


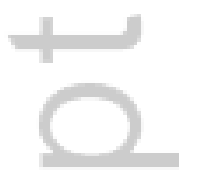




\section{REFERENCES}

1 Yannuzzi LA, Sorenson J, Spaide RF, Lipson B. Idiopathic Polypoidal Choroidal Vasculopathy (IPCV). Retina 1990; 10: 1-8.

2 Cheung CMG, Lai TYY, Ruamviboonsuk P et al. Polypoidal Choroidal Vasculopathy: Definition, Pathogenesis, Diagnosis, and Management. Ophthalmology 2018; 125: 708-724.

3 Spaide RF, Yannuzzi LA, Slakter JS, Sorenson J, Orlach DA. Indocyanine Green Videoangiography of Idiopathic Polypoidal Choroidal Vasculopathy. Retina 1995; 15: 100-110.

$4 \quad$ Li Y, You QS, Wei WB et al. Polypoidal choroidal vasculopathy in adult Chinese: The Beijing eye study. Ophthalmology 2014; 121: 2290-2291.

5 Uyama M, Wada M, Nagai Y et al. Polypoidal choroidal vasculopathy: Natural history. Am J Ophthalmo/ 2002; 133: 639-648.

6 Alasil T, Munoz N, Keane PA et al. Characteristics and racial variations of polypoidal choroidal vasculopathy in tertiary centers in the United States and United Kingdom. Int J Retin Vitr 2017; 3: 1-11.

7 Byeon SH, Lew YJ, Lee SC, Kwon OW. Clinical features and follow-up results of pulsating polypoidal choroidal vasculopathy treated with photodynamic therapy. Acta Ophthalmo/ 2010; 88: 660-668.

8 Cho JH, Ryoo NK, Cho KH et al. Incidence Rate of Massive Submacular Hemorrhage and its Risk Factors in Polypoidal Choroidal Vasculopathy. Am J Ophthalmo/ 2016; 169: 79-88.

9 Fine HF, Iranmanesh R, Del Priore LV et al. Surgical outcomes after massive subretinal hemorrhage secondary to age-related macular degeneration. Retina 2010; 30: 1588-1594.

10 Koh A, Lai TYY, Takahashi K et al. Efficacy and Safety of Ranibizumab With or Without Verteporfin Photodynamic Therapy for Polypoidal Choroidal 
Vasculopathy. JAMA Ophthalmo/2017; 248649.

doi:10.1001/jamaophthalmol.2017.4030.

11 Lee WK, Iida T, Ogura $Y$ et al. Efficacy and Safety of Intravitreal Aflibercept for Polypoidal Choroidal Vasculopathy in the PLANET Study. JAMA Ophthalmol 2018; 136: 786.

12 Kang HG, Kang $\mathrm{H}$, Byeon SH et al. Long-term visual outcomes for treatment of submacular haemorrhage secondary to polypoidal choroidal vasculopathy. Clin Exp Ophthalmo/2018. doi:10.1111/ceo.13198.

13 Shin JY, Lee J min, Byeon SH. Anti-vascular endothelial growth factor with or without pneumatic displacement for submacular hemorrhage. Am J Ophthalmo/2015; 159: 904-914.

14 Kitahashi M, Baba T, Sakurai M et al. Pneumatic displacement with intravitreal bevacizumab for massive submacular hemorrhage due to polypoidal choroidal vasculopathy. Clin Ophthalmol 2014; 8: 485-491.

15 De Jong JH, Van Zeeburg EJT, Cereda MG et al. Intravitreal versus Subretinal administration of recombinant tissue Plasminogen activator combined with gas for acute submacular hemorrhages due to age-related macular degeneration. Retina 2016; 36: 914-925. 


\section{University Library}

\section{- M M I E E R VA A gateway to Melbourne's research publications}

Minerva Access is the Institutional Repository of The University of Melbourne

Author/s:

Cugley, DR;Fagan, $X$

Title:

The needle, or the knife?

Date:

2018-11

Citation:

Cugley, D. R. \& Fagan, X. (2018). The needle, or the knife?. Clin Exp Ophthalmol, 46 (8), pp.851-853. https://doi.org/10.1111/ceo.13413.

Persistent Link:

http://hdl.handle.net/11343/284817 